\title{
A refinement of the integral form of Jensen's inequality
}

\section{László Horváth}

\section{${ }^{*}$ Correspondence:}

Ihorvath@almos.uni-pannon.hu

Department of Mathematics,

University of Pannonia, Egyetem u.

10, Veszprém, 8200, Hungary

\begin{abstract}
There are a lot of refinements of the discrete Jensen's inequality, and this problem has been studied by many authors. It is also a natural problem to give analogous results for the classical Jensen's inequality. In spite of this, few papers have been published dealing with this problem. The purpose of this paper is to give some refinements of the classical Jensen's inequality. The results give a new approach of this topic. Moreover, new discrete inequalities can be derived, and the integral analogous of discrete inequalities can be obtained. We also have new refinements of the left-hand side of the Hermite-Hadamard inequality.
\end{abstract}

MSC: 26D07; 26A51

Keywords: Jensen's inequality; convex function; measure space; Hermite-Hadamard inequality

\section{Introduction}

In view of applications in different parts of mathematics, the classical Jensen's inequality is especially noteworthy, as well as useful.

Theorem A (see [2]) Let fbe an integrable function on a probability space $(X, \mathcal{A}, P)$ taking values in an interval $I \subset \mathbb{R}$. Then $\int_{X} f d P$ lies in I. If $q$ is a convex function on I such that $q \circ f$ is P-integrable, then

$$
q\left(\int_{X} f d P\right) \leq \int_{X} q \circ f d P
$$

The discrete version of the Jensen's inequality is also particularly important.

Theorem B (see [8]) Let $C$ be a convex subset of a real vector space $V$, and let $q: C \rightarrow$ $\mathbb{R}$ be a convex function. If $p_{1}, \ldots, p_{n}$ are nonnegative numbers with $p_{1}+\cdots+p_{n}=1$, and $v_{1}, \ldots, v_{n} \in C$, then

$$
q\left(\sum_{i=1}^{n} p_{i} v_{i}\right) \leq \sum_{i=1}^{n} p_{i} q\left(v_{i}\right) .
$$

Various attempts have been made by many authors to refine the discrete Jensen's inequality (2). Basic papers in this direction were [10] and [9]. [6] and [4] contain essential

\section{Springer}

(c) 2012 Horváth; licensee Springer. This is an Open Access article distributed under the terms of the Creative Commons Attribution License (http://creativecommons.org/licenses/by/2.0), which permits unrestricted use, distribution, and reproduction in any medium, provided the original work is properly cited. 
generalizations of some earlier results. Some other types of refinements have been studied in the recent papers [12] and [5]. The treatment of the problem is similar in all the mentioned and other papers: Create an expression $A=A\left(q, v_{i}, p_{i}\right)$ satisfying the inequality

$$
q\left(\sum_{i=1}^{n} p_{i} v_{i}\right) \leq A \leq \sum_{i=1}^{n} p_{i} q\left(v_{i}\right),
$$

where $A$ is a sum whose index set is a subset (mainly a proper subset) of either $\{1, \ldots, n\}^{k}$ or $\{1, \ldots, k\}^{n}$ for some $k \in\{1,2, \ldots\}$. For further details, see [5].

It is also a natural problem to give analogous results for the classical Jensen's inequality (1). In spite of this, few papers have been published dealing with this problem (see [3] and [11]). One important reason is indicated: It is not possible to use the same machinery that is used in the discrete case. To the author's best knowledge, it does not exist any refinements of (1) in the form

$$
q\left(\int_{X} f d P\right) \leq B \leq \int_{X} q \circ f d P
$$

where $B=B(q, f, P)$ is an integral over a proper subset of $X^{k}$ for some $k \in\{1,2, \ldots\}$.

In this paper, such a refinement of (1) is obtained. The results not only extend and generalize earlier results, but give a new method to refine inequality (1). Moreover, the integral versions of classical discrete inequalities can be obtained. The inspiration for our result comes from the approach applied in [6]. We give such a version which shows the new feature clearly, but the method can be extended.

\section{Main results}

For the results from integration theory, see [2].

We consider the following conditions:

$\left(\mathrm{C}_{1}\right)$ Let $(X, \mathcal{A}, \mu)$ be a $\sigma$-finite measure space such that $\mu(X)>0$.

The integrable functions are considered to be measurable.

$\left(\mathrm{C}_{2}\right)$ Let $\varphi$ be a positive function on $X$ such that $\int_{X} \varphi d \mu=1$.

In this case, the measure $P$ defined on $\mathcal{A}$ by

$$
P(A):=\int_{A} \varphi d \mu
$$

is a probability measure having density $\varphi$ with respect to $\mu$. An $\mathcal{A}$-measurable function $g: X \rightarrow \mathbb{R}$ is $P$-integrable if and only if $g \varphi$ is $\mu$-integrable, and the relationship between the $P$ - and $\mu$-integrals is

$$
\int_{X} g d P=\int_{X} g \varphi d \mu
$$

$\left(\mathrm{C}_{3}\right)$ Let $k \geq 2$ be a fixed integer.

The $\sigma$-algebra in $X^{k}$ generated by the projection mappings $p r_{m}: X^{k} \rightarrow X(m=1, \ldots, k)$

$$
p r_{m}\left(x_{1}, \ldots, x_{k}\right):=x_{m}
$$


is denoted by $\mathcal{A}^{k} . \mu^{k}$ means the product measure on $\mathcal{A}^{k}$ : This measure is uniquely ( $\mu$ is $\sigma$-finite) specified by

$$
\mu^{k}\left(A_{1} \times \cdots \times A_{k}\right):=\mu\left(A_{1}\right) \cdots \mu\left(A_{k}\right), \quad A_{m} \in \mathcal{A}, m=1, \ldots, k .
$$

We shall also use the following projection mappings: For $m=1, \ldots, k$ define $p r^{m}: X^{k} \rightarrow$ $X^{k-1}$ by

$$
\operatorname{pr}^{m}\left(x_{1}, \ldots, x_{k}\right):=\left(x_{1}, \ldots, x_{m-1}, x_{m+1}, \ldots, x_{k}\right)
$$

For every $Q \in \mathcal{A}^{k}$ and for all $m=1, \ldots, k$, the sets

$$
\begin{aligned}
Q_{m, x}:= & \left\{\left(x_{1}, \ldots, x_{m-1}, x_{m+1}, \ldots, x_{k}\right) \in X^{k-1} \mid\right. \\
& \left.\left(x_{1}, \ldots, x_{m-1}, x, x_{m+1}, \ldots, x_{k}\right) \in Q\right\}
\end{aligned}
$$

and

$$
Q_{x_{1}, \ldots, x_{m-1}, x_{m+1}, \ldots, x_{k}}:=\left\{x \in X \mid\left(x_{1}, \ldots, x_{m-1}, x, x_{m+1}, \ldots, x_{k}\right) \in Q\right\}
$$

are called $x$-sections of $Q(x \in X)$ and $\left(x_{1}, \ldots, x_{m-1}, x_{m+1}, \ldots, x_{k}\right)$-sections of $Q\left(\left(x_{1}, \ldots\right.\right.$, $\left.\left.x_{m-1}, x_{m+1}, \ldots, x_{k}\right) \in X^{k-1}\right)$, respectively. We note that the sets $Q_{m, x}$ lie in $\mathcal{A}^{k-1}$, while $Q_{x_{1}, \ldots, x_{m-1}, x_{m+1}, \ldots, x_{k}} \in \mathcal{A}$.

$\left(\mathrm{C}_{4}\right)$ Let $S \in \mathcal{A}^{k}$ such that

$$
p r_{m}(S) \in \mathcal{A}, \quad m=1, \ldots, k \text { and } \bigcup_{m=1}^{k} p r_{m}(S)=X,
$$

and

$$
\left.l(x):=\sum_{m=1}^{k} \beta_{m} \mu^{k-1}\left(S_{m, x}\right) \in\right] 0, \infty[, \quad x \in X,
$$

where $\beta_{1}, \ldots, \beta_{k}$ are fixed positive numbers.

We stress that the first condition in (4) is necessary. For example, there exists a Borel set in $\mathbb{R}^{2}$ whose image under the first projection map is not a Borel set in $\mathbb{R}$ (see [7]). The function $l$ is $\mathcal{A}$-measurable.

Under the conditions $\left(C_{1}\right)-\left(C_{4}\right)$, we introduce the functions $\left.\psi: X^{k} \rightarrow\right] 0, \infty[$

$$
\psi\left(x_{1}, \ldots, x_{k}\right):=\sum_{j=1}^{k} \frac{\beta_{j} \varphi\left(x_{j}\right)}{l\left(x_{j}\right)}
$$

and $\left.\psi^{i}: X^{k-1} \rightarrow\right] 0, \infty[(i=1, \ldots, k)$

$$
\psi^{i}\left(x_{1}, \ldots, x_{i-1}, x_{i+1}, \ldots, x_{k}\right):=\sum_{\substack{j=1 \\ j \neq i}}^{k} \frac{\beta_{j} \varphi\left(x_{j}\right)}{l\left(x_{j}\right)}
$$


Since

$$
\psi=\sum_{j=1}^{k} \frac{\beta_{j}\left(\varphi \circ p r_{j}\right)}{l \circ p r_{j}}
$$

$\psi$ is $\mathcal{A}^{k}$-measurable. Similarly, $\psi^{i}(i=1, \ldots, k)$ is $\mathcal{A}^{k-1}$-measurable.

$\left(C_{5}\right)$ Suppose $\operatorname{pr}^{m}(S) \in \mathcal{A}^{k-1}(m=1, \ldots, k)$.

Now we formulate the main results.

Theorem 1 Assume $\left(\mathrm{C}_{1}\right)-\left(\mathrm{C}_{4}\right)$. Let $f: X \rightarrow \mathbb{R}$ be a P-integrable function taking values in an interval $I \subset \mathbb{R}$, and let $q$ be a convex function on $I$ such that $q \circ f$ is P-integrable. Then

(a)

$$
\begin{aligned}
q\left(\int_{X} f d P\right) & =q\left(\int_{X} f \varphi d \mu\right) \leq N_{k} \\
& :=\int_{S} q\left(\frac{1}{\psi\left(x_{1}, \ldots, x_{k}\right)} \sum_{j=1}^{k} \frac{\beta_{j} \varphi\left(x_{j}\right)}{l\left(x_{j}\right)} f\left(x_{j}\right)\right) \psi\left(x_{1}, \ldots, x_{k}\right) d \mu^{k}\left(x_{1}, \ldots, x_{k}\right) \\
& \leq \int_{X}(q \circ f) \varphi d \mu=\int_{X} q \circ f d P .
\end{aligned}
$$

(b) If $\left(\mathrm{C}_{5}\right)$ is also satisfied, then

$$
\begin{aligned}
q\left(\int_{X} f d P\right)= & q\left(\int_{X} f \varphi d \mu\right) \leq N_{k} \leq N_{k-1} \\
:= & \frac{1}{k-1} \sum_{i=1}^{k} \int_{p r^{i}(S)} \mu\left(S_{x_{1}, \ldots, x_{i-1}, x_{i+1}, \ldots, x_{k}}\right) \\
& \times q\left(\frac{1}{\psi^{i}\left(x_{1}, \ldots, x_{i-1}, x_{i+1}, \ldots, x_{k}\right)} \sum_{j=1}^{k} \frac{\beta_{j} \varphi\left(x_{j}\right)}{l\left(x_{j}\right)} f\left(x_{j}\right)\right) \\
& \times \psi^{i}\left(x_{1}, \ldots, x_{i-1}, x_{i+1}, \ldots, x_{k}\right) d \mu^{k-1}\left(x_{1}, \ldots, x_{i-1}, x_{i+1}, \ldots, x_{k}\right) \\
\leq & \int_{X}(q \circ f) \varphi d \mu=\int_{X} q \circ f d P .
\end{aligned}
$$

By applying the method used in the proof of the preceding theorem, it is possible to obtain a chain of refinements of the form

$$
q\left(\int_{X} f \varphi d \mu\right) \leq N_{k} \leq N_{k-1} \leq \cdots \leq N_{2} \leq N_{1}=\int_{X}(q \circ f) \varphi d \mu,
$$

but some measurability problems crop up and it is not so easy to construct the expressions $N_{i}(i=k-2, \ldots, 2)$. These difficulties disappear entirely if $S:=X$. In this case, we have the following theorem.

Theorem 2 Assume $\left(\mathrm{C}_{1}\right)-\left(\mathrm{C}_{3}\right)$, and let $\mu(X)<\infty$. If $f: X \rightarrow \mathbb{R}$ is a P-integrable function taking values in an interval $I \subset \mathbb{R}$, and $q$ is a convex function on $I$ such that $q \circ f$ 
is P-integrable, then

$$
\begin{aligned}
q\left(\int_{X} f d P\right) & =q\left(\int_{X} f \varphi d \mu\right) \\
& \leq \cdots \leq N_{k} \leq \cdots \leq N_{2} \leq N_{1}=\int_{X}(q \circ f) \varphi d \mu=\int_{X} q \circ f d P, \quad k \geq 1
\end{aligned}
$$

where

$$
N_{k}:=\frac{1}{k \mu(X)^{k-1}} \int_{X^{k}} q\left(\frac{\sum_{j=1}^{k} \varphi\left(x_{j}\right) f\left(x_{j}\right)}{\sum_{j=1}^{k} \varphi\left(x_{j}\right)}\right) \sum_{j=1}^{k} \varphi\left(x_{j}\right) d \mu^{k}\left(x_{1}, \ldots, x_{k}\right), \quad k \geq 1 .
$$

\section{Discussion and applications}

The following special situations show the force of our results: They extend and generalize some earlier results; new refinements of the discrete Jensen's inequality can be constructed; the integral version of known discrete inequalities can be derived.

1. Suppose $(X, \mathcal{A}, \mu)$ is a probability space, $\varphi(x):=1(x \in X), S:=X^{k}$, and $\sum_{m=1}^{k} \beta_{m}=1$. Then $\left(C_{1}\right)-\left(C_{5}\right)$ are satisfied. Suppose also that $f: X \rightarrow \mathbb{R}$ is a $\mu$-integrable function taking values in an interval $I \subset \mathbb{R}$, and $q$ is a convex function on $I$ such that $q \circ f$ is $\mu$-integrable. In this case, Theorem 1(a) gives Theorem 1.3(a) in [3]:

$$
q\left(\int_{X} f d \mu\right) \leq \int_{X^{k}} q\left(\sum_{j=1}^{k} \beta_{j} f\left(x_{j}\right)\right) d \mu^{k}\left(x_{1}, \ldots, x_{k}\right) \leq \int_{X}(q \circ f) d \mu
$$

If $\beta_{m}=\frac{1}{k}(m=1, \ldots, k)$ also holds, then Theorem 1.3(b) in [3] comes from Theorem 1(b):

$$
\begin{aligned}
& \int_{X^{k+1}} q\left(\frac{1}{k+1} \sum_{j=1}^{k+1} f\left(x_{j}\right)\right) d \mu^{k+1}\left(x_{1}, \ldots, x_{k+1}\right) \\
& \leq \int_{X^{k}} q\left(\frac{1}{k} \sum_{j=1}^{k} f\left(x_{j}\right)\right) d \mu^{k}\left(x_{1}, \ldots, x_{k}\right), \quad k \geq 1 .
\end{aligned}
$$

We can see that Theorem 1 is much more general than (5) even if $\mu$ is a probability measure. Moreover, Theorem 1(b) makes it possible to obtain a chain of refinements in (5):

$$
\begin{aligned}
q\left(\int_{X} f d \mu\right) \leq & \int_{X^{k}} q\left(\sum_{j=1}^{k} \beta_{j} f\left(x_{j}\right)\right) d \mu^{k}\left(x_{1}, \ldots, x_{k}\right) \\
\leq & \frac{1}{(k-1)} \sum_{i=1}^{k}\left(1-\beta_{i}\right) \\
& \times \int_{X^{k-1}} q\left(\frac{1}{1-\beta_{i}} \sum_{\substack{j=1 \\
j \neq i}}^{k} \beta_{j} f\left(x_{j}\right)\right) d \mu^{k-1}\left(x_{1}, \ldots, x_{i-1}, x_{i+1}, \ldots, x_{k}\right) \\
\leq & \int_{X}(q \circ f) d \mu .
\end{aligned}
$$


2. Let $X:=[a, b] \subset \mathbb{R}(a<b)$. The set of the Borel subsets of $\mathbb{R}\left(\mathbb{R}^{k}\right)$ is denoted by $\mathcal{B}\left(\mathcal{B}^{k}\right)$. $\lambda$ means the Lebesgue measure on $\mathcal{B}$. Let $q:[a, b] \rightarrow \mathbb{R}$ be a convex function.

The classical Hermite-Hadamard inequality (see [1]) says

$$
q\left(\frac{a+b}{2}\right) \leq \frac{1}{b-a} \int_{a}^{b} q(x) d x \leq \frac{q(a)+q(b)}{2} .
$$

We can obtain the following refinement of the left-hand side of the Hermite-Hadamard inequality.

Corollary 3 Let $S \subset[a, b]^{k}$ be a Borel set such that

$$
p r_{m}(S) \in \mathcal{B}, \quad m=1, \ldots, k \quad \text { and } \bigcup_{m=1}^{k} p r_{m}(S)=[a, b]
$$

and

$$
l(x)=\sum_{m=1}^{k} \beta_{m} \lambda^{k-1}\left(S_{m, x}\right)>0, \quad x \in[a, b]
$$

where $\beta_{m}>0(m=1, \ldots, k)$.

Then

$$
\begin{aligned}
q\left(\frac{a+b}{2}\right) & \leq \int_{S} q\left(\frac{1}{(b-a) \psi\left(x_{1}, \ldots, x_{k}\right)} \sum_{j=1}^{k} \frac{\beta_{j} x_{j}}{l\left(x_{j}\right)}\right) \psi\left(x_{1}, \ldots, x_{k}\right) d \mu^{k}\left(x_{1}, \ldots, x_{k}\right) \\
& \leq \frac{1}{b-a} \int_{a}^{b} q(x) d x .
\end{aligned}
$$

Proof We can apply Theorem 1 (a) to the pair of functions $\varphi, f:[a, b] \rightarrow \mathbb{R}, \varphi(x)=\frac{1}{b-a}$, and $f(x)=x$.

If $S=[a, b]^{k}$ and $\sum_{m=1}^{k} \beta_{m}=1$, then we have from (7) and Theorem 1(b) one of the main results in [13] as a special case:

$$
\begin{aligned}
q\left(\frac{a+b}{2}\right) \leq & \frac{1}{(b-a)^{k}} \int_{[a, b]^{k}} q\left(\sum_{j=1}^{k} \beta_{j} x_{j}\right) d \lambda^{k}\left(x_{1}, \ldots, x_{k}\right) \\
\leq & \sum_{i=1}^{k} \frac{1-\beta_{i}}{(k-1)(b-a)^{k-1}} \\
& \times \int_{[a, b]^{k-1}} q\left(\frac{1}{1-\beta_{i}} \sum_{\substack{j=1 \\
j \neq i}}^{k} \beta_{j} x_{j}\right) d \lambda^{k-1}\left(x_{1}, \ldots, x_{i-1}, x_{i+1}, \ldots, x_{k}\right) \\
\leq & \frac{1}{b-a} \int_{a}^{b} q(x) d x .
\end{aligned}
$$

Another concrete example can be constructed for (7) by using Corollary 5. 
3. In the following results, we consider noteworthy proper subsets of $\mathbb{R}^{k}$.

(a) Let $z, w \in \mathbb{R}, z<w$, and $m \geq 1$ be an integer. The simplex $S_{z, w}^{m}$ is defined by

$$
S_{z, w}^{m}:=\left\{\left(x_{1}, \ldots, x_{m}\right) \in \mathbb{R}^{m} \mid z \leq x_{1} \leq \cdots \leq x_{m} \leq w\right\} .
$$

Let $X:=[a, b] \subset \mathbb{R}(a<b)$, and $\mu$ be a finite measure on the trace $\sigma$-algebra $[a, b] \cap \mathcal{B}$ such that $\mu([a, b])>0$. Suppose $\varphi:[a, b] \rightarrow \mathbb{R}$ is a positive function such that $\int_{[a, b]} \varphi d \mu=1$. Fix an integer $k \geq 2$, and let $\beta_{m}>0(m=1, \ldots, k)$.

Choose $S:=S_{a, b}^{k}$. Then

$$
S_{1, x}=S_{x, b}^{k-1}, \quad S_{k, x}=S_{a, x}^{k-1}, \quad S_{m, x}=S_{a, x}^{m-1} \times S_{x, b}^{k-m}, \quad 2 \leq m \leq k-1,
$$

once the appropriate identification of $\mathbb{R}^{k-1}$ with $\mathbb{R}^{j-1} \times \mathbb{R}^{k-j}(2 \leq j \leq k-1)$ has been made. Therefore,

$$
l(x)=\sum_{m=1}^{k} \beta_{m} \mu^{m-1}\left(S_{a, x}^{m-1}\right) \mu^{k-m}\left(S_{x, b}^{k-m}\right)>0, \quad x \in[a, b],
$$

where $\mu^{0}\left(S_{a, x}^{0}\right)=\mu^{0}\left(S_{x, b}^{0}\right):=1$. Thus,

$$
\psi\left(x_{1}, \ldots, x_{k}\right)=\sum_{j=1}^{k} \frac{\beta_{j} \varphi\left(x_{j}\right)}{\sum_{m=1}^{k} \beta_{m} \mu^{m-1}\left(S_{a, x_{j}}^{m-1}\right) \mu^{k-m}\left(S_{x_{j}, b}^{k-m}\right)}, \quad\left(x_{1}, \ldots, x_{k}\right) \in[a, b]^{k}
$$

We can see that under the above assumptions $\left(\mathrm{C}_{1}\right)-\left(\mathrm{C}_{4}\right)$ are satisfied, so Theorem 1 can be applied.

Corollary 4 If $f:[a, b] \rightarrow \mathbb{R}$ is a P-integrable function taking values in an interval $I \subset \mathbb{R}$, and $q$ is a convex function on I such that $q \circ f$ is P-integrable, then

$$
\begin{aligned}
& q\left(\int_{[a, b]} f d P\right) \\
& =q\left(\int_{[a, b]} f \varphi d \mu\right) \\
& \quad \leq \int_{S} q\left(\frac{1}{\sum_{j=1}^{k} \frac{\beta_{j} \varphi\left(x_{j}\right)}{\sum_{m=1}^{k} \beta_{m} \mu^{m-1}\left(S_{a, x_{j}}^{m-1}\right) \mu^{k-m}\left(S_{x_{j}, b}^{k-m}\right)}} \sum_{j=1}^{k} \frac{\beta_{j} \varphi\left(x_{j}\right) f\left(x_{j}\right)}{\sum_{m=1}^{k} \beta_{m} \mu^{m-1}\left(S_{a, x_{j}}^{m-1}\right) \mu^{k-m}\left(S_{x_{j}, b}^{k-m}\right)}\right) \\
& \quad \times \sum_{j=1}^{k} \frac{\beta_{j} \varphi\left(x_{j}\right)}{\sum_{m=1}^{k} \beta_{m} \mu^{m-1}\left(S_{a, x_{j}}^{m-1}\right) \mu^{k-m}\left(S_{x_{j}, b}^{k-m}\right)} d \mu^{k}\left(x_{1}, \ldots, x_{k}\right) \\
& \leq \int_{[a, b]}(q \circ f) \varphi d \mu=\int_{[a, b]} q \circ f d P .
\end{aligned}
$$

Specifically, if $\mu=\lambda$, we have

$$
l(x)=\frac{1}{(k-1) !} \sum_{m=1}^{k} \beta_{m}\left(\begin{array}{c}
k-1 \\
m-1
\end{array}\right)\left(x_{j}-a\right)^{m-1}\left(b-x_{j}\right)^{k-m}, \quad x \in[a, b] .
$$


When $\beta_{1}=\cdots=\beta_{k}=\beta$, this says

$$
l(x)=\frac{\beta}{(k-1) !}(b-a)^{k-1}, \quad x \in[a, b],
$$

and in this case we have the inequality for the cube $[a, b]^{k}$

$$
\begin{aligned}
& q\left(\int_{a}^{b} f \varphi d \lambda\right) \\
& \quad \leq \frac{(k-1) !}{(b-a)^{k-1}} \int_{S} q\left(\frac{1}{\sum_{j=1}^{k} \varphi\left(x_{j}\right)} \sum_{j=1}^{k} \varphi\left(x_{j}\right) f\left(x_{j}\right)\right) \sum_{j=1}^{k} \varphi\left(x_{j}\right) d \lambda^{k}\left(x_{1}, \ldots, x_{k}\right) \\
& \quad=\frac{1}{k(b-a)^{k-1}} \int_{[a, b]^{k}} q\left(\frac{1}{\sum_{j=1}^{k} \varphi\left(x_{j}\right)} \sum_{j=1}^{k} \varphi\left(x_{j}\right) f\left(x_{j}\right)\right) \sum_{j=1}^{k} \varphi\left(x_{j}\right) d \lambda^{k}\left(x_{1}, \ldots, x_{k}\right) \\
& \quad \leq \int_{a}^{b}(q \circ f) \varphi d \lambda .
\end{aligned}
$$

Next, we show that inequality (8) extends the following well-known discrete inequality to an integral form. Similar results are quite rare in the literature (see [3]).

Theorem $\mathbf{C}$ (see [9]) Let I be an interval in $\mathbb{R}$, and let $q: I \rightarrow \mathbb{R}$ be a convex function. If $v_{1}, \ldots, v_{n} \in I$, then for each $k \geq 1$

$$
q\left(\frac{1}{n} \sum_{i=1}^{n} v_{i}\right) \leq \frac{1}{\left(\begin{array}{c}
n+k-1 \\
k
\end{array}\right)} \sum_{1 \leq i_{1} \leq \cdots \leq i_{k} \leq n} q\left(\frac{v_{i_{1}}+\cdots+v_{i_{k}}}{k}\right) \leq \frac{1}{n} \sum_{i=1}^{n} q\left(v_{i}\right) .
$$

Let $X:=[1, n] \subset \mathbb{R}(n \geq 1$ is an integer), and let $\mu$ be the measure on the trace $\sigma$-algebra $[1, n] \cap \mathcal{B}$ defined by $\mu:=\sum_{m=1}^{n} \frac{1}{n} \varepsilon_{m}$, where $\varepsilon_{m}$ is the unit mass at $m(m=1, \ldots, n)$. Suppose $\varphi(x):=1(x \in[1, n]), k \geq 2$ is a fixed integer, and $\beta_{m}=1(m=1, \ldots, k)$.

Some easy combinatorial considerations yield that for every $x \in[1, n]$ and $m=1, \ldots, k$

$$
\mu^{k-1}\left(S_{m, x}\right)=\frac{1}{n^{k-1}}\left(\begin{array}{c}
{[x]+m-2} \\
m-1
\end{array}\right)\left(\begin{array}{c}
n-[x]+k-m \\
k-m
\end{array}\right),
$$

where $[x]$ is the largest natural number that does not exceed $x$. Therefore,

$$
l(x)=\frac{1}{n^{k-1}}\left(\begin{array}{c}
n+k-1 \\
k-1
\end{array}\right), \quad x \in[1, n]
$$

Now, if $I$ is an interval in $\mathbb{R}, q: I \rightarrow \mathbb{R}$ is a convex function, and $f:[1, n] \rightarrow \mathbb{R}$ defined by

$$
f(i):= \begin{cases}v_{i}, & i=1, \ldots, n \\ 0, & \text { elsewhere }\end{cases}
$$

then (9) follows immediately from (8).

(b) Let $m \geq 1$ be an integer, $z \in \mathbb{R}^{m}$, and $r>0$. The open ball of radius $r$ centered at the point $z$ is denoted by $B_{m}(z, r)$. 
Consider the measure space (]$a, b[, \mathcal{B}, \lambda)(a<b)$. Suppose $\varphi:] a, b[\rightarrow \mathbb{R}$ is a positive function such that $\int_{a}^{b} \varphi d \lambda=1$. Fix an integer $k \geq 2$, and let $\beta_{m}>0(m=1, \ldots, k)$. Choose

$$
S:=B_{k}\left(\left(\frac{a+b}{2}, \ldots, \frac{a+b}{2}\right), \frac{b-a}{2}\right) .
$$

Then for all $x \in] a, b[$ and $m=1, \ldots, k$

$$
S_{m, x}=B_{k-1}\left(\left(\frac{a+b}{2}, \ldots, \frac{a+b}{2}\right), \sqrt{(b-x)(x-a)}\right) .
$$

Consequently,

$$
\left.l(x)=k \frac{2^{k-1}}{(k-1) ! !}\left(\frac{\pi}{2}\right)^{\left[\frac{k-1}{2}\right]}((b-x)(x-a))^{\frac{k-1}{2}}, \quad x \in\right] a, b[.
$$

According to this, for all $\left.\left(x_{1}, \ldots, x_{k}\right) \in\right] a, b\left[^{k}\right.$,

$$
\psi\left(x_{1}, \ldots, x_{k}\right)=\frac{1}{k \frac{2^{k-1}}{(k-1) ! !}\left(\frac{\pi}{2}\right)^{\left[\frac{k-1}{2}\right]}} \sum_{j=1}^{k} \frac{\beta_{j} \varphi\left(x_{j}\right)}{\sum_{m=1}^{k} \beta_{m}\left(\left(b-x_{j}\right)\left(x_{j}-a\right)\right)^{\frac{k-1}{2}}} .
$$

It is not hard to check that $\left(\mathrm{C}_{1}\right)-\left(\mathrm{C}_{4}\right)$ are satisfied in this situation, and thus Theorem 1 says:

Corollary 5 If $:] a, b[\rightarrow \mathbb{R}$ is a P-integrable function taking values in an interval $I \subset \mathbb{R}$, and $q$ is a convex function on I such that $q \circ f$ is P-integrable, then

$$
\begin{aligned}
& q\left(\int_{] a, b[} f d P\right) \\
& =q\left(\int_{a}^{b} f \varphi d \lambda\right) \leq \frac{1}{\left.k \frac{2^{k-1}(k-1) !}{2}\right)^{\left[\frac{k-1}{2}\right]}} \\
& \quad \times \int_{S} q\left(\frac{1}{\sum_{j=1}^{k} \frac{\beta_{j} \varphi\left(x_{j}\right)}{\sum_{m=1}^{k} \beta_{m}\left(\left(b-x_{j}\right)\left(x_{j}-a\right)\right)^{\frac{k-1}{2}}}} \sum_{j=1}^{k} \frac{\beta_{j} \varphi\left(x_{j}\right) f\left(x_{j}\right)}{\sum_{m=1}^{k} \beta_{m}\left(\left(b-x_{j}\right)\left(x_{j}-a\right)\right)^{\frac{k-1}{2}}}\right) \\
& \quad \times \sum_{j=1}^{k} \frac{\beta_{j} \varphi\left(x_{j}\right)}{\sum_{m=1}^{k} \beta_{m}\left(\left(b-x_{j}\right)\left(x_{j}-a\right)\right)^{\frac{k-1}{2}}} d \lambda^{k}\left(x_{1}, \ldots, x_{k}\right) \\
& \leq \int_{a}^{b}(q \circ f) \varphi d \lambda=\int_{] a, b[} q \circ f d P .
\end{aligned}
$$

By applying this result $\left(\varphi, f:[a, b] \rightarrow \mathbb{R}, \varphi(x)=\frac{1}{b-a}\right.$ and $\left.f(x)=x\right)$, we can have a special case of the refinement of the left-hand side of the Hermite-Hadamard inequality in (7).

4. We turn now to the case where $X$ is a countable set.

$\left(C_{1}^{1}\right)$ Consider the measure space $\left(X, 2^{X}, \mu\right)$, where either $X:=\{1, \ldots, n\}$ for some positive integer $n$ or $X:=\{0,1, \ldots\}, 2^{X}$ denotes the power set of $X$, and $\mu(\{u\}):=\mu_{u}$ is a positive number for all $u \in X$.

$\left(C_{2}^{1}\right)$ Let $\left(p_{u}\right)_{u \in X}$ be a sequence of positive numbers for which $\sum_{u \in X} p_{u} \mu_{u}=1$. 
$\left(C_{3}^{1}\right)$ Let $k \geq 2$ be a fixed integer.

We define the functions $\alpha_{v}^{j}(v \in X, j=1, \ldots, k)$ on $X^{k}$ by

$$
\alpha_{v}^{j}\left(u_{1}, \ldots, u_{k}\right):= \begin{cases}1, & \text { if } u_{j}=v \\ 0, & \text { if } u_{j} \neq v\end{cases}
$$

Then $\sum_{j=1}^{k} \alpha_{v}^{j}\left(u_{1}, \ldots, u_{k}\right)$ means the number of occurrences of $v$ in $\left(u_{1}, \ldots, u_{k}\right) \in X^{k}$. If $S \subset$ $X^{k}$, we introduce the following sums:

$$
\alpha_{S, v}^{j}:=\sum_{\left(u_{1}, \ldots, u_{k}\right) \in S} \alpha_{v}^{j}\left(u_{1}, \ldots, u_{k}\right), \quad v \in X, j=1, \ldots, k
$$

and

$$
\alpha_{S, v}:=\sum_{j=1}^{k} \alpha_{S, v}^{j}, \quad v \in X
$$

Every sum is either a nonnegative integer or $\infty$.

$\left(C_{4}^{1}\right)$ Let $S \subset X^{k}$ such that $\alpha_{S, v} \geq 1$ for all $v \in X$, and

$$
l(u):=\sum_{m=1}^{k} \beta_{m} \mu^{k-1}\left(S_{m, u}\right)<\infty, \quad u \in X
$$

where $\beta_{m}>0(m=1, \ldots, k)$.

Since $\mu_{u}>0$ for all $u \in X, l(u)>0$ for all $u \in X$. By the definition of the measure $\mu$,

$$
l(u)=\sum_{m=1}^{k} \beta_{m} \sum_{\left(u_{1}, \ldots, u_{j-1}, u, u_{j+1}, \ldots, u_{k}\right) \in S} \mu_{u_{1}} \cdots \mu_{u_{j-1}} \mu_{u_{j+1}} \cdots \mu_{u_{k}}, \quad u \in X .
$$

In this case, the function $\psi$ has the form

$$
\left.\psi: X^{k} \rightarrow\right] 0, \infty\left[, \quad \psi\left(u_{1}, \ldots, u_{k}\right):=\sum_{j=1}^{k} \frac{\beta_{j} p_{u_{j}}}{l\left(u_{j}\right)}\right.
$$

Now Theorem 1(a) can be formulated in the following way.

Corollary 6 Assume $\left(\mathrm{C}_{1}^{1}\right)-\left(\mathrm{C}_{4}^{1}\right)$, and let $\left(f_{u}\right)_{u \in X}$ be a sequence taking values in an interval $I \subset \mathbb{R}$ such that $\sum_{u \in X}\left|f_{u}\right| p_{u} \mu_{u}<\infty$. If $q$ is a convex function on $I$ such that $\sum_{u \in X}\left|q\left(f_{u}\right)\right| p_{u} \mu_{u}<\infty$, then

$$
\begin{aligned}
& q\left(\sum_{u \in X} f_{u} p_{u} \mu_{u}\right) \\
& \quad \leq \sum_{\left(u_{1}, \ldots, u_{k}\right) \in S} q\left(\frac{1}{\psi\left(u_{1}, \ldots, u_{k}\right)} \sum_{j=1}^{k} \frac{\beta_{j} p_{u_{j}}}{l\left(u_{j}\right)} f_{u_{j}}\right) \psi\left(u_{1}, \ldots, u_{k}\right) \mu_{u_{1}} \cdots \mu_{u_{k}} \\
& \quad \leq \sum_{u \in X} q\left(f_{u}\right) p_{u} \mu_{u} .
\end{aligned}
$$


Assume $\left(C_{1}^{1}\right)-\left(C_{4}^{1}\right)$, and suppose $\mu$ is the counting measure on $P(X)$, that is, $\mu_{u}:=1$ for all $u \in X$. For a set $A \in 2^{X}$, let $|A|$ denote the number of elements of $A$. Then $\sum_{u \in X} p_{u}=1$,

$$
l(u)=\sum_{m=1}^{k} \beta_{m} \alpha_{S, u}^{m}, \quad u \in X
$$

and

$$
\left.\psi: X^{k} \rightarrow\right] 0, \infty\left[, \quad \psi\left(u_{1}, \ldots, u_{k}\right):=\sum_{j=1}^{k} \frac{\beta_{j} p_{u_{j}}}{\sum_{m=1}^{k} \beta_{m} \alpha_{S, u_{j}}^{m}}\right.
$$

We note explicitly this particular case of Corollary 6.

Corollary 7 Assume $\left(C_{1}^{1}\right)-\left(C_{4}^{1}\right)$, where $\mu$ is the counting measure on $2^{X}$, and let $\left(f_{u}\right)_{u \in X}$ be a sequence taking values in an interval $I \subset \mathbb{R}$ such that $\sum_{u \in X}\left|f_{u}\right| p_{u}<\infty$. If $q$ is a convex function on I such that $\sum_{u \in X}\left|q\left(f_{u}\right)\right| p_{u}<\infty$, then

$$
\begin{aligned}
& q\left(\sum_{u \in X} f_{u} p_{u}\right) \\
& \quad \leq \sum_{\left(u_{1}, \ldots, u_{k}\right) \in S}\left(\sum_{j=1}^{k} \frac{\beta_{j} p_{u_{j}}}{\sum_{m=1}^{k} \beta_{m} \alpha_{S, u_{j}}^{m}}\right) q\left(\frac{1}{\sum_{j=1}^{k} \frac{\beta_{j} p_{u_{j}}}{\sum_{m=1}^{k} \beta_{m} \alpha_{S, u_{j}}^{m}}} \sum_{j=1}^{k} \frac{\beta_{j} p_{u_{j}}}{\sum_{m=1}^{k} \beta_{m} \alpha_{S, u_{j}}^{m}} f_{u_{j}}\right) \\
& \quad \leq \sum_{u \in X} q\left(f_{u}\right) p_{u} .
\end{aligned}
$$

Corollary 6 corresponds to Theorem 2 in [4], but in [4] only finite sets are considered. If $X=\{1, \ldots, n\}$ and $\beta_{m}=1(m=1, \ldots, k)$, then Theorem 1(a) in [6] contains Corollary 7, but Corollary 6 makes sense in a lot of other cases (for example, for countably infinite sets).

Next, some examples are given.

The first example deals with a relatively flexile case.

Example 8 (a) Assume $\left(\mathrm{C}_{1}\right)-\left(\mathrm{C}_{3}\right)$, and let $A_{m} \in \mathcal{A}(m=1, \ldots, k)$ such that $0<\mu\left(A_{m}\right)<\infty$ $(m=1, \ldots, k)$ and $\bigcup_{m=1}^{k} A_{m}=X$. Define $S:=A_{1} \times \cdots \times A_{k}$. Then (4) holds and

$$
l(x)=\left(\prod_{m=1}^{k} \mu\left(A_{m}\right)\right) \sum_{m=1}^{k}\left(\frac{\beta_{m} \chi_{m}(x)}{\mu\left(A_{m}\right)}\right), \quad x \in X
$$

where $\beta_{m}>0(m=1, \ldots, k)$, and $\chi_{m}: X \rightarrow \mathbb{R}$ means the characteristic function of $A_{m}(m=$ $1, \ldots, k)$. We can see that $\left(C_{4}\right)$ is satisfied and

$$
\psi\left(x_{1}, \ldots, x_{k}\right)=\frac{1}{\left(\prod_{m=1}^{k} \mu\left(A_{m}\right)\right)} \sum_{j=1}^{k} \frac{\beta_{j} \varphi\left(x_{j}\right)}{\sum_{m=1}^{k}\left(\frac{\beta_{m} \chi_{m}\left(x_{j}\right)}{\mu\left(A_{m}\right)}\right)}, \quad\left(x_{1}, \ldots, x_{k}\right) \in X^{k}
$$

The condition $\left(C_{5}\right)$ is also true, since

$$
\operatorname{pr}^{m}(S)=A_{1} \times \cdots \times A_{m-1} \times A_{m+1} \times \cdots \times A_{k}, \quad m=1, \ldots, k
$$


Moreover, for $m=1, \ldots, k$,

$$
\begin{aligned}
& S_{x_{1}, \ldots, x_{m-1}, x_{m+1}, \ldots, x_{k}} \\
& \quad= \begin{cases}A_{m}, & \text { if }\left(x_{1}, \ldots, x_{m-1}, x_{m+1}, \ldots, x_{k}\right) \in A_{1} \times \cdots \times A_{m-1} \times A_{m+1} \times \cdots \times A_{k}, \\
\varnothing, & \text { otherwise. }\end{cases}
\end{aligned}
$$

It follows that Theorem 1 can be applied.

(b) We consider the special case of (a), when the sets $A_{m}(m=1, \ldots, k)$ are pairwise disjoint (a special partition of $X$ ). Let the function $\tau$ be defined on $X$ by

$$
\tau(x):=m, \quad \text { if } x \in A_{m} .
$$

Then

$$
l(x)=\left(\prod_{m=1}^{k} \mu\left(A_{m}\right)\right) \frac{\beta_{\tau(x)}}{\mu\left(A_{\tau(x)}\right)}, \quad x \in X
$$

and

$$
\psi\left(x_{1}, \ldots, x_{k}\right)=\frac{1}{\left(\prod_{m=1}^{k} \mu\left(A_{m}\right)\right)} \sum_{j=1}^{k} \frac{\beta_{j} \varphi\left(x_{j}\right) \mu\left(A_{\tau\left(x_{j}\right)}\right)}{\beta_{\tau\left(x_{j}\right)}}, \quad\left(x_{1}, \ldots, x_{k}\right) \in X^{k}
$$

The second example corresponds to Corollary 6.

Example 9 Let $X:=\{0,1, \ldots\}$, let $\left(p_{u}\right)_{u=0}^{\infty}$ be a sequence of positive numbers for which $\sum_{u=0}^{\infty} p_{u}=1$, and let $\left(f_{u}\right)_{u=0}^{\infty}$ be a sequence taking values in an interval $I \subset \mathbb{R}$ such that $\sum_{u=0}^{\infty}\left|f_{u}\right| p_{u}<\infty$. Define

$$
S:=\left\{\left(u_{1}, u_{2}\right) \in X^{2} \mid u_{1} \leq u_{2} \leq 2 u_{1}\right\}
$$

An easy calculation shows that $l(u)=\alpha_{S, u}=u+\left[\frac{u}{2}\right]+2(\geq 2)$ for all $u \in X$, where $\left[\frac{u}{2}\right]$ denotes the greatest integer that does not exceed $\frac{u}{2}$. If $q$ is a convex function on $I$ such that $\sum_{u=0}^{\infty}\left|q\left(f_{u}\right)\right| p_{u}<\infty$, then by Corollary 7

$$
\begin{aligned}
q\left(\sum_{u=0}^{\infty} f_{u} p_{u}\right) \leq & \sum_{u=0}^{\infty}\left(\sum_{v=u}^{2 u}\left(\frac{p_{u}}{u+\left[\frac{u}{2}\right]+2}+\frac{p_{v}}{v+\left[\frac{v}{2}\right]+2}\right)\right. \\
& \left.\times q\left(\frac{\frac{p_{u}}{u+\left[\frac{u}{2}\right]+2} f_{u}+\frac{p_{v}}{v+\left[\frac{v}{2}\right]+2} f_{v}}{\frac{p_{u}}{u+\left[\frac{u}{2}\right]+2}+\frac{p_{v}}{v+\left[\frac{v}{2}\right]+2}}\right)\right) \leq \sum_{u=0}^{\infty} q\left(f_{u}\right) p_{u} .
\end{aligned}
$$

The final example illustrates the case $X:=\mathbb{R}$.

Example 10 Consider the measure space $(\mathbb{R}, \mathcal{B}, \lambda)$. The function $\varphi: \mathbb{R} \rightarrow \mathbb{R}, \varphi(x)=$ $\frac{1}{\sqrt{2 \pi}} e^{-x^{2} / 2}$ is the density of the standard normal distribution on $\mathbb{R}$, and thus $\int_{-\infty}^{\infty} \varphi=1$. Let

$$
S:=\left\{(x, y) \in \mathbb{R}^{2} \mid x-1 \leq y \leq x+1\right\}
$$


Then $\left(C_{1}\right)-\left(C_{3}\right)$ are satisfied. Let $f: \mathbb{R} \rightarrow \mathbb{R}$ be a Borel measurable function taking values in an interval $I \subset \mathbb{R}$ such that $f \varphi$ is integrable, and let $q$ be a convex function on $I$ such that $(q \circ f) \varphi$ is integrable. By Theorem 1(a),

$$
\begin{aligned}
& q\left(\int_{-\infty}^{\infty} f \varphi\right) \\
& \quad \leq \frac{1}{4 \sqrt{2 \pi}} \int_{-\infty}^{\infty}\left(\int_{x-1}^{x+1} q\left(\frac{e^{-x^{2} / 2} f(x)+e^{-y^{2} / 2} f(y)}{e^{-x^{2} / 2}+e^{-y^{2} / 2}}\right)\left(e^{-x^{2} / 2}+e^{-y^{2} / 2}\right) d y\right) d x \\
& \quad \leq \int_{-\infty}^{\infty}(q \circ f) \varphi .
\end{aligned}
$$

\section{Preliminary results and the proof of the main result}

We first establish a result which will be fundamental to our treatment.

Lemma 11 Assume $\left(\mathrm{C}_{1}\right)-\left(\mathrm{C}_{4}\right)$, and let $f: X \rightarrow \mathbb{R}$ be a P-integrable function. Then

$$
\int_{X} f d P=\int_{X} f \varphi d \mu=\int_{S}\left(\sum_{j=1}^{k} \frac{\beta_{j} \varphi\left(x_{j}\right)}{l\left(x_{j}\right)} f\left(x_{j}\right)\right) d \mu^{k}\left(x_{1}, \ldots, x_{k}\right) .
$$

Proof The functions

$$
\frac{\beta_{j}\left(\varphi \circ p r_{j}\right)}{l \circ p r_{j}} f \circ p r_{j}, \quad j=1, \ldots, k
$$

are obviously $\mathcal{A}^{k}$-measurable on $S$.

Suppose first that the function $f$ is nonnegative. By $\left(C_{3}\right), p r_{j}(S) \in \mathcal{A}$, and hence the theorem of Fubini implies that

$$
\begin{aligned}
& \int_{S} \frac{\beta_{j} \varphi\left(x_{j}\right)}{l\left(x_{j}\right)} f\left(x_{j}\right) d \mu^{k}\left(x_{1}, \ldots, x_{k}\right) \\
& \quad=\int_{p r_{j}(S)}\left(\int_{S_{j}, x_{j}} \frac{\beta_{j} \varphi\left(x_{j}\right)}{l\left(x_{j}\right)} f\left(x_{j}\right) d \mu^{k-1}\left(x_{1}, \ldots, x_{j-1}, x_{j+1}, \ldots, x_{k}\right)\right) d \mu\left(x_{j}\right) \\
& \quad=\int_{p r_{j}(S)} \frac{\beta_{j} \varphi\left(x_{j}\right)}{l\left(x_{j}\right)} f\left(x_{j}\right) \mu^{k-1}\left(S_{j, x_{j}}\right) d \mu\left(x_{j}\right), \quad j=1, \ldots, k .
\end{aligned}
$$

It follows from (10) that

$$
\begin{aligned}
& \int_{S}\left(\sum_{j=1}^{k} \frac{\beta_{j} \varphi\left(x_{j}\right)}{l\left(x_{j}\right)} f\left(x_{j}\right)\right) d \mu^{k}\left(x_{1}, \ldots, x_{k}\right) \\
& =\sum_{j=1}^{k} \int_{p r_{j}(S)} \frac{\beta_{j} \varphi\left(x_{j}\right)}{l\left(x_{j}\right)} f\left(x_{j}\right) \mu^{k-1}\left(S_{j, x_{j}}\right) d \mu\left(x_{j}\right) .
\end{aligned}
$$

If $\left(i_{1}, \ldots, i_{k}\right) \in\{0,1\}^{k}$, then let

$$
A_{i_{1}, \ldots, i_{k}}:=\bigcap_{m=1}^{k} p r_{m}(S)^{\left(i_{m}\right)},
$$


where

$$
p r_{m}(S)^{\left(i_{m}\right)}:=\left\{\begin{array}{ll}
p r_{m}(S), & \text { if } i_{m}=1, \\
X \backslash p r_{m}(S), & \text { if } i_{m}=0,
\end{array} \quad m=1, \ldots, k\right.
$$

The sets $A_{i_{1}, \ldots, i_{k}}\left(\left(i_{1}, \ldots, i_{k}\right) \in\{0,1\}^{k}\right)$ are pairwise disjoint and measurable. Moreover, by (4),

$$
\bigcup_{\left(i_{1}, \ldots, i_{k}\right) \in\{0,1\}^{k}} A_{i_{1}, \ldots, i_{k}}=\bigcup_{m=1}^{k} p r_{m}(S)=X .
$$

These establishments with (11) imply that

$$
\begin{aligned}
& \int_{S}\left(\sum_{j=1}^{k} \frac{\beta_{j} \varphi\left(x_{j}\right)}{l\left(x_{j}\right)} f\left(x_{j}\right)\right) d \mu^{k}\left(x_{1}, \ldots, x_{k}\right) \\
& =\sum_{\left(i_{1}, \ldots, i_{k}\right) \in\{0,1\}^{k}} \int_{A_{i_{1}, \ldots, i_{k}}}\left(\frac{\varphi(x)}{l(x)} f(x) \sum_{\substack{m \in\{1, \ldots, k\} \\
i_{m}=1}} \beta_{m} \mu^{k-1}\left(S_{m, x}\right)\right) d \mu(x) .
\end{aligned}
$$

Choose $\left(i_{1}, \ldots, i_{k}\right) \in\{0,1\}^{k}$. It is clear that $S_{m, x}=\varnothing$ if $x \in A_{i_{1}, \ldots, i_{k}}$ and $i_{m}=0$, and hence

$$
\sum_{\substack{m \in\{1, \ldots, k\} \\ i_{m}=1}} \beta_{m} \mu^{k-1}\left(S_{m, x}\right)=l(x), \quad x \in A_{i_{1}, \ldots, i_{k}} .
$$

Therefore, (12) gives

$$
\begin{aligned}
& \int_{S}\left(\sum_{j=1}^{k} \frac{\beta_{j} \varphi\left(x_{j}\right)}{l\left(x_{j}\right)} f\left(x_{j}\right)\right) d \mu^{k}\left(x_{1}, \ldots, x_{k}\right) \\
& =\sum_{\left(i_{1}, \ldots, i_{k}\right) \in\{0,1\}^{k}} \int_{A_{i_{1}, \ldots, i_{k}}} \varphi f d \mu=\int_{X} f \varphi d \mu .
\end{aligned}
$$

Having disposed of the nonnegativity of the function $f$, we have from the first part of the proof that

$$
\int_{X}|f| d P=\int_{X}|f| \varphi d \mu=\int_{S}\left(\sum_{j=1}^{k} \frac{\beta_{j} \varphi\left(x_{j}\right)}{l\left(x_{j}\right)}\left|f\left(x_{j}\right)\right|\right) d \mu^{k}\left(x_{1}, \ldots, x_{k}\right),
$$

and, therefore, the functions

$$
\frac{\beta_{j}\left(\varphi \circ p r_{j}\right)}{l \circ p r_{j}} f \circ p r_{j}, \quad j=1, \ldots, k
$$

are $\mu^{k}$-integrable over $S$. By using this, the result follows by an argument entirely similar to that for the nonnegative case.

The proof is complete. 
Remark 12 Under the conditions of Lemma 11, we have

(a) The functions

$$
\frac{\beta_{j}\left(\varphi \circ p r_{j}\right)}{l \circ p r_{j}} f \circ p r_{j}, \quad j=1, \ldots, k
$$

are $\mu^{k}$-integrable over $S$.

(b) The measure $P_{k}$ defined on $S \cap \mathcal{A}^{k}$ by

$$
P_{k}(A):=\int_{A} \psi d \mu^{k}=\int_{A}\left(\sum_{j=1}^{k} \frac{\beta_{j} \varphi\left(x_{j}\right)}{l\left(x_{j}\right)}\right) d \mu^{k}\left(x_{1}, \ldots, x_{k}\right)
$$

is a probability measure.

Lemma 13 Assume $\left(\mathrm{C}_{1}\right)-\left(\mathrm{C}_{4}\right)$. Let $f: X \rightarrow \mathbb{R}$ be a P-integrable function taking values in an interval $I \subset \mathbb{R}$, and let $q$ be a convex function on $I$ such that $q \circ f$ is $P$-integrable.

(a) The function

$$
g:=\left(q \circ\left(\frac{1}{\psi} \sum_{j=1}^{k} \frac{\beta_{j}\left(\varphi \circ p r_{j}\right)}{l \circ p r_{j}}\left(f \circ p r_{j}\right)\right)\right) \psi
$$

is $\mu^{k}$-integrable over $S$.

(b) The functions

$$
h_{i}:=\left(q \circ\left(\frac{1}{\psi^{i} \circ p r^{i}} \sum_{\substack{j=1 \\ j \neq i}}^{k} \frac{\beta_{j}\left(\varphi \circ p r_{j}\right)}{l \circ p r_{j}}\left(f \circ p r_{j}\right)\right)\right)\left(\psi^{i} \circ p r^{i}\right), \quad i=1, \ldots, k
$$

are $\mu^{k}$-integrable over $S$.

Proof (a) It is easy to check that for fixed $\left(x_{1}, \ldots, x_{k}\right) \in S$

$$
\frac{1}{\psi\left(x_{1}, \ldots, x_{k}\right)} \frac{\beta_{j} \varphi\left(x_{j}\right)}{l\left(x_{j}\right)}, \quad j=1, \ldots, k
$$

are positive numbers with

$$
\frac{1}{\psi\left(x_{1}, \ldots, x_{k}\right)} \sum_{j=1}^{k} \frac{\beta_{j} \varphi\left(x_{j}\right)}{l\left(x_{j}\right)}=1
$$

This gives immediately that for every $\left(x_{1}, \ldots, x_{k}\right) \in S$

$$
\frac{1}{\psi\left(x_{1}, \ldots, x_{k}\right)} \sum_{j=1}^{k} \frac{\beta_{j} \varphi\left(x_{j}\right)}{l\left(x_{j}\right)} f\left(x_{j}\right) \in I
$$

and, therefore, by Theorem B,

$$
g\left(x_{1}, \ldots, x_{k}\right) \leq \sum_{j=1}^{k} \frac{\beta_{j} \varphi\left(x_{j}\right)}{l\left(x_{j}\right)} q\left(f\left(x_{j}\right)\right), \quad\left(x_{1}, \ldots, x_{k}\right) \in S
$$


Since the function $q$ is convex on $I$, it is lower semicontinuous on $I$ and, therefore, the function $g$ is $\mathcal{A}^{k}$-measurable.

Choose an interior point $a$ of $I$. The convexity of $q$ on $I$ implies that

$$
q(t) \geq q(a)+q_{+}^{\prime}(a)(t-a), \quad t \in I
$$

where $q_{+}^{\prime}(a)$ means the right-hand derivative of $q$ at $a$. It follows from this and from (13) that

$$
\begin{gathered}
q(a) \psi\left(x_{1}, \ldots, x_{k}\right)+q_{+}^{\prime}(a)\left(\sum_{j=1}^{k} \frac{\beta_{j} \varphi\left(x_{j}\right)}{l\left(x_{j}\right)} f\left(x_{j}\right)-a \psi\left(x_{1}, \ldots, x_{k}\right)\right) \\
\leq g\left(x_{1}, \ldots, x_{k}\right) \leq \sum_{j=1}^{k} \frac{\beta_{j} \varphi\left(x_{j}\right)}{l\left(x_{j}\right)} q\left(f\left(x_{j}\right)\right), \quad\left(x_{1}, \ldots, x_{k}\right) \in S .
\end{gathered}
$$

Now we can apply Remark 12(a), by the $P$-integrability of the functions $1_{X}, f$ and $q \circ f$.

(b) Fix $i$ from the set $\{1, \ldots, k\}$. We can prove as in (a) by using the $\mathcal{A}^{k}$-measurability of $h_{i}$ and the estimates

$$
\begin{aligned}
& q(a) \psi^{i}\left(x_{1}, \ldots, x_{i-1}, x_{i+1}, \ldots, x_{k}\right) \\
& \quad+q_{+}^{\prime}(a)\left(\sum_{\substack{j=1 \\
j \neq i}}^{k} \frac{\beta_{j} \varphi\left(x_{j}\right)}{l\left(x_{j}\right)} f\left(x_{j}\right)-a \psi^{i}\left(x_{1}, \ldots, x_{i-1}, x_{i+1}, \ldots, x_{k}\right)\right) \\
& \leq h_{i}\left(x_{1}, \ldots, x_{k}\right) \leq \sum_{\substack{j=1 \\
j \neq i}}^{k} \frac{\beta_{j} \varphi\left(x_{j}\right)}{l\left(x_{j}\right)} q\left(f\left(x_{j}\right)\right), \quad\left(x_{1}, \ldots, x_{k}\right) \in S .
\end{aligned}
$$

The proof is complete.

Now we are able to prove the main results.

Proof of Theorem 1 (a) By Lemma 11,

$$
\begin{aligned}
q\left(\int_{X} f d P\right) & =q\left(\int_{X} f \varphi d \mu\right) \\
& =q\left(\int_{S}\left(\sum_{j=1}^{k} \frac{\beta_{j} \varphi\left(x_{j}\right)}{l\left(x_{j}\right)} f\left(x_{j}\right)\right) d \mu^{k}\left(x_{1}, \ldots, x_{k}\right)\right) \\
& =q\left(\int_{S}\left(\frac{1}{\psi\left(x_{1}, \ldots, x_{k}\right)} \sum_{j=1}^{k} \frac{\beta_{j} \varphi\left(x_{j}\right)}{l\left(x_{j}\right)} f\left(x_{j}\right) \psi\left(x_{1}, \ldots, x_{k}\right)\right) d \mu^{k}\left(x_{1}, \ldots, x_{k}\right)\right) \\
& =q\left(\int_{S}\left(\frac{1}{\psi\left(x_{1}, \ldots, x_{k}\right)} \sum_{j=1}^{k} \frac{\beta_{j} \varphi\left(x_{j}\right)}{l\left(x_{j}\right)} f\left(x_{j}\right)\right) d P_{k}\left(x_{1}, \ldots, x_{k}\right)\right) .
\end{aligned}
$$


Since $P_{k}$ is a probability measure on $S \cap \mathcal{A}^{k}$, it follows from the previous part, Theorem A, Lemma 11, and Lemma 13(a) that

$$
\begin{aligned}
q\left(\int_{X} f d P\right) & =q\left(\int_{X} f \varphi d \mu\right) \\
& \leq \int_{S} q\left(\frac{1}{\psi\left(x_{1}, \ldots, x_{k}\right)} \sum_{j=1}^{k} \frac{\beta_{j} \varphi\left(x_{j}\right)}{l\left(x_{j}\right)} f\left(x_{j}\right)\right) d P_{k}\left(x_{1}, \ldots, x_{k}\right) \\
& =\int_{S} q\left(\frac{1}{\psi\left(x_{1}, \ldots, x_{k}\right)} \sum_{j=1}^{k} \frac{\beta_{j} \varphi\left(x_{j}\right)}{l\left(x_{j}\right)} f\left(x_{j}\right)\right) \psi\left(x_{1}, \ldots, x_{k}\right) d \mu^{k}\left(x_{1}, \ldots, x_{k}\right) \\
& \leq \int_{S}\left(\sum_{j=1}^{k} \frac{\beta_{j} \varphi\left(x_{j}\right)}{l\left(x_{j}\right)} q\left(f\left(x_{j}\right)\right)\right) d \mu^{k}=\int_{X}(q \circ f) \varphi d \mu=\int_{X} q \circ f d P .
\end{aligned}
$$

Now (a) has been proven.

(b) By using the convexity of $q$, an easy manipulation leads to

$$
\begin{aligned}
& q\left(\frac{1}{\psi\left(x_{1}, \ldots, x_{k}\right)} \sum_{j=1}^{k} \frac{\beta_{j} \varphi\left(x_{j}\right)}{l\left(x_{j}\right)} f\left(x_{j}\right)\right) \\
& =q\left(\sum_{i=1}^{k} \frac{\sum_{\substack{j=1 \\
j \neq i}}^{k} \frac{\beta_{j} \varphi\left(x_{j}\right)}{l\left(x_{j}\right)} f\left(x_{j}\right)}{\psi^{i}\left(x_{1}, \ldots, x_{i-1}, x_{i+1}, \ldots, x_{k}\right)} \frac{\psi^{i}\left(x_{1}, \ldots, x_{i-1}, x_{i+1}, \ldots, x_{k}\right)}{(k-1) \psi\left(x_{1}, \ldots, x_{k}\right)}\right) \\
& \leq \frac{1}{(k-1) \psi\left(x_{1}, \ldots, x_{k}\right)} \sum_{i=1}^{k} \psi^{i}\left(x_{1}, \ldots, x_{i-1}, x_{i+1}, \ldots, x_{k}\right) \\
& \quad \times q\left(\frac{1}{\psi^{i}\left(x_{1}, \ldots, x_{i-1}, x_{i+1}, \ldots, x_{k}\right)} \sum_{\substack{j=1 \\
j \neq i}}^{k} \frac{\beta_{j} \varphi\left(x_{j}\right)}{l\left(x_{j}\right)} f\left(x_{j}\right)\right) \\
& \leq \frac{1}{(k-1) \psi\left(x_{1}, \ldots, x_{k}\right)} \sum_{i=1}^{k}\left(\sum_{\substack{j=1 \\
j \neq i}}^{k} \frac{\beta_{j} \varphi\left(x_{j}\right)}{l\left(x_{j}\right)} q\left(f\left(x_{j}\right)\right)\right) \\
& =\frac{1}{\psi\left(x_{1}, \ldots, x_{k}\right)} \sum_{j=1}^{k} \frac{\beta_{j} \varphi\left(x_{j}\right)}{l\left(x_{j}\right)} q\left(f\left(x_{j}\right)\right), \quad\left(x_{1}, \ldots, x_{k}\right) \in S .
\end{aligned}
$$

Consequently, by applying $\left(\mathrm{C}_{5}\right)$, Lemma $13(\mathrm{~b})$, and the theorem of Fubini, we have

$$
\begin{aligned}
N_{k}= & \int_{S} q\left(\frac{1}{\psi\left(x_{1}, \ldots, x_{k}\right)} \sum_{j=1}^{k} \frac{\beta_{j} \varphi\left(x_{j}\right)}{l\left(x_{j}\right)} f\left(x_{j}\right)\right) \psi\left(x_{1}, \ldots, x_{k}\right) d \mu^{k}\left(x_{1}, \ldots, x_{k}\right) \\
\leq & \frac{1}{k-1} \sum_{i=1}^{k} \int_{S} q\left(\frac{1}{\psi^{i}\left(x_{1}, \ldots, x_{i-1}, x_{i+1}, \ldots, x_{k}\right)} \sum_{\substack{j=1 \\
j \neq i}}^{k} \frac{\beta_{j} \varphi\left(x_{j}\right)}{l\left(x_{j}\right)} f\left(x_{j}\right)\right) \\
& \times \psi^{i}\left(x_{1}, \ldots, x_{i-1}, x_{i+1}, \ldots, x_{k}\right) d \mu^{k}\left(x_{1}, \ldots, x_{k}\right)
\end{aligned}
$$




$$
\begin{aligned}
= & \frac{1}{k-1} \sum_{i=1}^{k} \int_{p r^{i}(S)}\left(\int _ { S _ { x _ { 1 } , \ldots , x _ { i - 1 } , x _ { i + 1 } , \ldots , x _ { k } } } q \left(\frac{1}{\psi^{i}\left(x_{1}, \ldots, x_{i-1}, x_{i+1}, \ldots, x_{k}\right)}\right.\right. \\
& \left.\left.\times \sum_{\substack{j=1 \\
j \neq i}}^{k} \frac{\beta_{j} \varphi\left(x_{j}\right)}{l\left(x_{j}\right)} f\left(x_{j}\right)\right) d \mu\left(x_{i}\right)\right) \\
& \times \psi^{i}\left(x_{1}, \ldots, x_{i-1}, x_{i+1}, \ldots, x_{k}\right) d \mu^{k-1}\left(x_{1}, \ldots, x_{i-1}, x_{i+1}, \ldots, x_{k}\right) \\
= & N_{k-1} \leq \int_{X}(q \circ f) \varphi d \mu .
\end{aligned}
$$

The proof is complete.

Proof of Theorem 2 Apply Theorem 1 with $S:=X^{k}$ and $\beta_{m}=1(m=1, \ldots, k)$. Then the conditions $\left(\mathrm{C}_{4}\right)$ (by using $\left.\mu(X)<\infty\right)$ and $\left(\mathrm{C}_{5}\right)$ are satisfied,

$$
\psi\left(x_{1}, \ldots, x_{k}\right):=\frac{1}{k \mu(X)^{k-1}} \sum_{j=1}^{k} \varphi\left(x_{j}\right), \quad\left(x_{1}, \ldots, x_{k}\right) \in S,
$$

and $\left.\psi^{i}: X^{k-1} \rightarrow\right] 0, \infty[(i=1, \ldots, k)$ has the form

$$
\psi^{i}\left(x_{1}, \ldots, x_{i-1}, x_{i+1}, \ldots, x_{k}\right):=\frac{1}{k \mu(X)^{k-1}} \sum_{\substack{j=1 \\ j \neq i}}^{k} \varphi\left(x_{j}\right) .
$$

Therefore,

$$
N_{k}=\frac{1}{k \mu(X)^{k-1}} \int_{X^{k}} q\left(\frac{\sum_{j=1}^{k} \varphi\left(x_{j}\right) f\left(x_{j}\right)}{\sum_{j=1}^{k} \varphi\left(x_{j}\right)}\right) \sum_{j=1}^{k} \varphi\left(x_{j}\right) d \mu^{k}\left(x_{1}, \ldots, x_{k}\right)
$$

and

$$
\begin{aligned}
N_{k-1}= & \frac{1}{k-1} \sum_{i=1}^{k} \int_{X^{k-1}}\left(\mu(X) q\left(\frac{\sum_{\substack{j=1 \\
j \neq i}}^{k} \frac{\varphi\left(x_{j}\right)}{l\left(x_{j}\right)} f\left(x_{j}\right)}{\sum_{\substack{j=1 \\
j \neq i}}^{k} \varphi\left(x_{j}\right)}\right)\right) \\
& \times \frac{1}{k \mu(X)^{k-1}} \sum_{\substack{j=1 \\
j \neq i}}^{k} \varphi\left(x_{j}\right) d \mu^{k-1}\left(x_{1}, \ldots, x_{i-1}, x_{i+1}, \ldots, x_{k}\right) \\
= & \frac{1}{(k-1) \mu(X)^{k-2}} \int_{X^{k-1}} q\left(\frac{\sum_{j=1}^{k-1} \varphi\left(x_{j}\right) f\left(x_{j}\right)}{\sum_{j=1}^{k-1} \varphi\left(x_{j}\right)}\right) \sum_{j=1}^{k-1} \varphi\left(x_{j}\right) d \mu^{k-1}\left(x_{1}, \ldots, x_{k-1}\right) .
\end{aligned}
$$

The proof is complete. 
Received: 24 May 2012 Accepted: 3 August 2012 Published: 16 August 2012

\section{References}

1. Hadamard, J: Étude sur les propriétés des fonctions entiéres en particulier d'une fonction considérée par Riemann. J. Math. Pures Appl. 58, 171-215 (1893)

2. Hewitt, E, Stromberg, KR: Real and Abstract Analysis. Graduate Text in Mathematics, vol. 25. Springer, Berlin (1965)

3. Horváth, L: Inequalities corresponding to the classical Jensen's inequality. J. Math. Inequal. 3(2), 189-200 (2009)

4. Horváth, L: A method to refine the discrete Jensen's inequality for convex and mid-convex functions. Math. Comput. Model. 54, 2451-2459 (2011)

5. Horváth, L: A parameter-dependent refinement of the discrete Jensen's inequality for convex and mid-convex functions. J. Inequal. Appl. 2011, 26 (2011)

6. Horváth, L, Pečarić, J: A refinement of the discrete Jensen's inequality. Math. Inequal. Appl. 14(4), 777-791 (2011)

7. Kechris, AS: Classical Descriptive Set Theory. Springer, Berlin (1995)

8. Mitrinović, DS, Pečarić, JE, Fink, AM: Classical and New Inequalities in Analysis. Kluwer Academic, Dordrecht (1993)

9. Pečarić, JE, Svrtan, D: Unified approach to refinements of Jensen's inequalities. Math. Inequal. Appl. 5, 45-47 (2002)

10. Pečarić, JE, Volenec, V: Interpolation of the Jensen inequality with some applications. Österr. Akad. Wiss. Math.-Naturw. Kl. Sitzungsber. II 197, 463-467 (1988)

11. Rooin, J: A refinement of Jensen's inequality. JIPAM. J. Inequal. Pure Appl. Math. 6(2), Article 38 (2005)

12. Xiao, Z-G, Srivastava, HM, Zhang, Z-H: Further refinements of the Jensen inequalities based upon samples with repetitions. Math. Comput. Model. 51, 592-600 (2010)

13. Yang, GS, Wang, CS: Some refinements of Hadamard's inequality. Tamkang J. Math. 28, 87-92 (1997)

doi:10.1186/1029-242X-2012-178

Cite this article as: Horváth: A refinement of the integral form of Jensen's inequality. Journal of Inequalities and Applications 2012 2012:178.

\section{Submit your manuscript to a SpringerOpen ${ }^{\circ}$ journal and benefit from:}

- Convenient online submission

Rigorous peer review

- Immediate publication on acceptance

- Open access: articles freely available online

- High visibility within the field

- Retaining the copyright to your article 